\title{
The relationship between the symptom of fatigue and the functioning of patients with inflammatory bowel diseases after surgery
}

\author{
Grażyna Bączyk ${ }^{1}$, Katarzyna A. Kozłowska ${ }^{1}$, Dorota Formanowicz², Ewelina Białas ${ }^{1}$, Jacek Karoń ${ }^{3}$, \\ Piotr Krokowicz ${ }^{3}$ \\ ${ }^{1}$ Department of Nursing, Faculty of Health Sciences, Poznan University of Medical Sciences, Poznan, Poland \\ 2Department of Clinical Biochemistry and Laboratory Medicine, Poznan University of Medical Sciences, Poznan, Poland \\ ${ }^{3}$ Department of General and Colorectal Surgery, Faculty of Health Sciences, Poznan University of Medical Sciences, Poznan, \\ Poland
}

Gastroenterology Rev 2019; 14 (4): 242-249

DOI: https://doi.org/10.5114/pg.2019.90251

Key words: inflammatory bowel disease, fatigue, functioning.

Address for correspondence: Grażyna Bączyk PhD, Department of Nursing, Faculty of Health Sciences, Poznan University of Medical Sciences, 11 Smoluchowskiego St, 60-179 Poznan, Poland, phone: +48 692562 364, e-mail: gbaczyk@ump.edu.pl

\begin{abstract}
Introduction: The occurrence of fatigue in patients suffering from inflammatory bowel diseases (IBD) is influenced by pain, frequent bowel movements, stress associated with symptoms and time of their occurrence reaction of surroundings, fear for their own health, sleep disturbances, side effects of pharmacological treatment, physical and mental exhaustion, hindered social contacts and difficulties at work.

Aim: To evaluate the fatigue and the assessment of functioning of patients with IBD, who were treated surgically.

Material and methods: To evaluate the functioning of patients, a Polish version of the Inflammatory Bowel Disease Questionnaire was used. To evaluate the occurrence of fatigue in studied subjects, a Polish version of the Functional Assessment of Chronic Illness Therapy - Fatigue Scale was used. The activity of disease was evaluated with the use of the Crohn's Disease Activity Index for patients with Crohn's disease (CD) and the Clinical Activity Index for patients with ulcerative colitis (UC).

Results: Before surgery, there was no significant difference between CD and UC patients, with regard to the mean FACIT-F (28.76 for CD and 28.76 for UC, $p=0.72$ ). Also, after surgery, there was no significant difference between CD and UC patients, with regard to the mean FACIT-F (14.8 for CD and 16.0 for UC, $p=0.71$ ). The IBD patients who underwent surgery for CD and UC had significantly lower FACIT-F scores compared to the patients before the surgery ( $p=0.001$ and $p=0.0001$, respectively). IBD patients who underwent surgery for CD and UC had significantly better functioning and higher IBDQ total scores compared to the patients before the surgery.

Conclusions: Surgical treatment significantly reduces the fatigue symptom in patients with IBD. The severity of fatigue correlates with disease activity and functioning in the respective areas.
\end{abstract}

\section{Introduction}

Crohn's disease (CD) and ulcerative colitis (UC) are the two main types of inflammatory bowel disease (IBD) of unknown aetiology. Both diseases are often similar in terms of clinical symptoms - they both affect digestive system, except that UC affects the large intestine and progresses proximally from the rectum, while CD occupies the small intestine (most frequently) and large intestine. Common symptoms of both diseases include frequent stomach aches, diarrhoea (often bloody in patients with UC), and weight loss. There are also many symptoms from outside of the digestive system, such as articular or skin changes [1]. The dominant symptom reported by patients is fatigue.

According to the Van Langenberg Systematic Review on fatigue in IBD patients, fatigue prevalence ranges from $41 \%$ to $48 \%$ in remission, and to $86 \%$ when the disease is active [2-4]. According to a recent study Grimstad et al., fatigue was found in nearly $50 \%$ of patients with newly diagnosed, active, and untreated IBD [5].

The Medical Library Subject Heading (MeSH) defines the symptom of fatigue as the state of weariness fol- 
lowing a period of exertion, mental or physical, characterised by a decreased capacity for work and reduced efficiency to respond to stimuli [6].

Fatigue is most frequently defined as a lack of energy to perform, an inability to concentrate, physical and mental exhaustion, and general reluctance. There is also fatigue defined as discouragement, apathy, indifference, and lack of vigour. Fatigue is also present in patients with chronic disease (cancer, neurological, rheumatic diseases, and chronic infectious diseases) [7, 8]. This symptom prevents normal functioning, both professional and social, as well as in daily/private life [9]. The consequences of fatigue also involve loneliness, helplessness, the appearance of cognitive dysfunction, and frustration [10]. Chronic fatigue can also determine decisions concerning having children [11]. The occurrence of fatigue in patients suffering from inflammatory bowel diseases before surgery is influenced by pain, frequent bowel movements, stress associated with symptoms and the time of their occurrence (e.g. during social meetings), reaction to surroundings, fear for one's health, sleep disturbances, side effects of pharmacological treatment, physical and mental exhaustion, hindered social contact, and difficulties at work (due to diarrhoea). Whereas after the surgical treatment the image of fatigue is influenced by complications, postoperative pain, fear of stoma care and response of the environment (especially family), and acceptance of the new situation [9-11].
However, a few months after surgery, patients achieve improvement in the functioning and quality of life. Improvement of the quality of life $(\mathrm{Q} O L)$ is the main determinant in decision-making for surgery in patients with UC and CD. Research has shown that surgical interventions positively affect QoL in patients with IBD $[9,12]$.

\section{Aim}

This study aimed to evaluate the fatigue and the assessment of the functioning of patients with IBD, who were treated surgically, and to determine the relationship between fatigue and functioning of patients, taking into account the activity of the disease.

\section{Material and methods}

The study involved 60 patients, 30 of them suffering from $C D$ and 30 from UC. All patients were treated surgically: 13 CD patients experienced treatment of anal fistulas, nine had bowel resection, and 8 had right-sided hemicolectomy. Thirty of the UC patients underwent proctocolectomy with temporary ileostomy.

All patients were treated at the Clinic and Department of General and Colorectal Surgery at Poznan University of Medical Sciences.

A demographic characteristic of the studied groups is presented in Table I.

Table I. Demographic and clinical characteristic of the patients

\begin{tabular}{|c|c|c|}
\hline Variables & Crohn's disease $(n=30)$ & Ulcerative colitis $(n=30)$ \\
\hline Age of patients, mean (SD) & $37.8(7.57)$ & $35.1(9.18)$ \\
\hline Women, $n(\%)$ & $18(60)$ & $14(46.7)$ \\
\hline Secondary and higher education, $n(\%)$ & $27(90)$ & $25(83.3)$ \\
\hline \multicolumn{3}{|l|}{ Crohn's Disease Activity Index (CDAI): } \\
\hline Mean (SD) (CDAI) before the surgery & $263(10.0)$ & \\
\hline Mean (SD) (CDAI) after the surgery & $145(9.6)$ & \\
\hline \multicolumn{3}{|l|}{ Clinical Activity Index (CAI): } \\
\hline Mean (SD) (CAI) before the surgery & & $8.2(2.6)$ \\
\hline Mean (SD); (CAI) after the surgery & & $6.8(3.1)$ \\
\hline Disease duration, mean (SD) [years] & $10.2(0.2)$ & $11.4(0.7)$ \\
\hline \multicolumn{3}{|l|}{ Treatment, $n(\%)$ : } \\
\hline Anal fistula treatment & $13(43.3)$ & \\
\hline Bowel resection & $9(30.0)$ & \\
\hline Right-sided hemicolectomy & $8(26.7)$ & \\
\hline Proctocolectomy with temporary ileostomy & & $30(100)$ \\
\hline
\end{tabular}


To evaluate the functioning of patients, a Polish version of the Inflammatory Bowel Disease Questionnaire (IBDQ) was used [13]. In order to use the above mentioned scale, a license was obtained (License Agreement - ORCIP file number: IBDQ07-032, McMaster University, Hamilton, Ontario, Canada).

IBDQ is used to evaluate the overall functioning (score range: 32-224) and in specific dimensions:

a) digestive tract symptoms (score range: 1-70): number of stools, loose stools and presence of blood in the stool, abdominal pain, intestinal cramps, abdominal distension, straining to have a bowel movement, soiling of underwear, nausea, and stomach ailments;

b) systemic function (score range: 1-35): fatigue and weariness, level of energy, malaise, sleep disturbances, weight loss;

c) emotional function (score range: 1-84): impatience, fear concerning necessity of surgical treatment, fear concerning inability of finding the toilet, dejection, anxiety, relaxation, embarrassment caused by intestinal problems, anger, irritability (or crying), depression, lack of understanding from other people, satisfaction with own life;

d) social function (score range: $1-35$ points): absence from work or school, necessity of cancelling social meetings, inability to play sports and to spend leisure time in a favourite way, avoidance of social meetings and places without direct access to the toilet, limited sexual activity.

Higher scores obtained by the respondents represent better life performance.

To evaluate the occurrence of fatigue in the studied subjects, the Polish version of the Functional Assessment of Chronic Illness Therapy - Fatigue Scale (Version 4) (FACIT-F) was used [14].

This mentioned scale is used to evaluate fatigue in patients with chronic diseases. It consists of 13 questions concerning fatigue occurring during the performance of daily living activities over the preceding week. The questions refer to physical activity during the day, interpersonal relationships, and the impact of fatigue on the psyche. The patient has five answers for choosing (from 0 (I am not tired) to 4 points (I am very tired)). A maximum of 52 points can be obtained; the higher the score, the higher the severity of fatigue.

The activity of disease was evaluated with the use of Crohn's Disease Activity Index (CDAI) for patients with $C D$ and the Clinical Activity Index (CAI) for patients with UC $[15,16]$.

The CDAI scale evaluates the following: frequency of loose stools, occurrence of stomach aches, presence of fistulas, joint inflammations, iris and choroid inflammation, ulcerative dermatitis, anal fistulas, persistent high temperature, weight loss, anti-diarrhoeal medications, and value of haematocrit. The maximum score that a patient can obtain is 600 . Scores not higher than 150 points indicate a mild course of disease, more than 150 points -active disease, and above 450 points - severe course of disease.

The CAI scale evaluates the following: number of bowel movements within the day and night, presence of blood in stool, faecal incontinence during the day and at night, painful bowel movements, and ant diarrheal. Symptoms are assessed in points, the higher the score, the more serious the disease. The range of scores from 0 to 21 (mild course of disease - from 0 to 10 points severe course of disease - above 10 points).

The study was conducted in the period between January 2013 and April 2015, twice: the day before the surgery and 3 months after.

This research was approved by the Ethical Committee of Poznan University of Medical Sciences (no. 126/07).

\section{Statistical analysis}

For the characteristics of the examined groups and variables, the following measures of descriptive statistics were used: mean, median, standard deviation, and percentage fractions. To evaluate the differences between distinguished groups the $T$-test was performed for unrelated variables, and the results were confirmed with Mann-Whitney nonparametric $U$ test.

Fisher's test was used when required for statistical analysis of differences in the levels of fatigue and functioning before and after the surgery. To assess the co-variability of examined variables the $r$-Pearson correlation coefficient was used. We considered $p<0.05$ as statistically significant.

\section{Results}

\section{Demographic and disease characteristics}

A total of 60 patients were studied, 30 of them suffering from $C D$ and 30 from UC. The mean age of the CD patients was $37.8 \pm 7.57$ years, and for UC patients $-35.1 \pm 9.18$ years. Sixty percent of CD patients and $46.7 \%$ of UC patients were women. The mean duration of disease was $10.2 \pm 0.2$ years for CD patients and $11.4 \pm 0.7$ years for UC patients. The mean CDAI before surgery for CD patients was $263 \pm 10.0$ points and the mean of CAI for UC patients was $8.2 \pm 2.6$ points. After surgery (145 \pm 9.6 and $6.8 \pm 3.1$, respectively).

All of the patients were treated surgically. Among CD patients: 13 CD patients experienced treatment of anal fistulas, 9 had bowel resection, and 8 had right-sided hemicolectomy. Thirty of the UC patients underwent proctocolectomy with temporary ileostomy. 


\section{The functioning of the of patients before the surgery}

The functioning of patients before the surgery was evaluated on the basis of the mean value of the IBDQ scale. We compared the means in specific areas of the scale for patients with CD and UC. Mean IBDQ scores of bowel symptoms, systemic, emotional, and social domains of the IBDQ are shown in Table II. Bowel symptoms, systemic symptoms, and emotional and social function domains had lower functioning scores for CD patients than for UC patients. There was a significant difference between CD patients and UC patients for systemic symptoms (CD 13.01 vs. UC 19.52), and emotional (CD 39.67 vs. UC 40.62) and social function (CD 12.05 vs. UC 21.12), $p<0.001$.

There was no significant difference in mean scores of bowel symptoms (CD 24.78 vs. UC 25.23, $p=0.142$ ). Also, there was no significant difference in mean scores of IBDQ total (CD 90.66 vs. UC 91.02, $p=0.234$ ).

\section{The functioning of the of patients after the surgery}

Mean IBDQ scores of bowel symptoms and systemic, emotional, and social domains of IBDQ after surgery are shown in Table III. There was significant difference between $C D$ patients and UC for bowel symptoms (CD 44.18 vs. UC 53.93, $p=0.007$ ), systemic symptoms (CD 22.11 vs. UC 27.59, $p<0.001$ ), emotional (CD 40.87 vs. UC 59.8, $p<0.001$ ), and social functions (CD 20.55 vs. UC 29.32, $p<0.001$ ). Also, there was a significant difference in mean scores of IBDQ total (CD 92.03 vs. UC 94.82, $p<0.01)$.

\section{Comparison of patients' functioning before and after the surgery}

A comparison of patients' functioning before and after the surgery are shown in Table IV. IBD patients who underwent surgery for CD and UC had significantly better functioning (bowel symptoms, systemic, emotional, social domains) and higher IBDQ total scores compared to the patients before the surgery.

\section{Fatigue symptoms before and after surgery}

Fatigue before and after surgery are shown in Table V. Before surgery there was no significant difference between $C D$ and UC patients with regard to the mean FACIT-F (28.76 for CD and 28.76 for UC, $p=0.72$ ). Also, after surgery there was no significant difference between $C D$ and UC patients with regard to the mean FACIT-F (14.8 for CD and 16.0 for UC, $p=0.71$ ).

IBD patients who underwent surgery for CD and UC had significantly lower FACIT-F scores compared to patients before the surgery $(p=0.001$ and $p=0.0001$, respectively).

Table II. Functioning of patients in areas of the IBDQ scale before surgery

\begin{tabular}{|c|c|c|c|}
\hline $\begin{array}{l}\text { Inflammatory Bowel Disease } \\
\text { Questionnaire }\end{array}$ & $\begin{array}{c}\text { Crohn's disease }(n=30) \\
\text { Mean (SD) }\end{array}$ & $\begin{array}{l}\text { Ulcerative colitis }(n=30) \\
\text { Mean (SD) }\end{array}$ & $P$-value \\
\hline Bowel symptoms (1-70) & $24.78(3.65)$ & $25.23(3.41)$ & 0.142 \\
\hline Systemic function (1-35) & $13.01(2.45)$ & $19.52(3.45)$ & $<0.001$ \\
\hline Emotional function (1-84) & $39.67(4.65)$ & $40.62(4.23)$ & $<0.001$ \\
\hline Social function (1-35) & $12.05(4.91)$ & $21.12(3.56)$ & $<0.001$ \\
\hline Total IBDQ (32-224) & $90.66(21.15)$ & $91.02(20.31)$ & 0.234 \\
\hline
\end{tabular}

The higher the scores obtained by the patients, the better the functioning.

Table III. Functioning of patients in areas of the IBDQ scale after surgery

\begin{tabular}{lll}
$\begin{array}{l}\text { Inflammatory Bowel Disease } \\
\text { Questionnaire }\end{array}$ & $\begin{array}{c}\text { Crohn's disease }(n=30) \\
\text { Mean (SD) }\end{array}$ & $\begin{array}{c}\text { Ulcerative colitis }(n=30) \\
\text { Mean (SD) }\end{array}$ \\
\hline Bowel symptoms (1-70) & $44.18(3.55)$ & $53.93(4.23)$ \\
\hline Systemic function (1-35) & $22.11(2.55)$ & $27.59(3.49)$ \\
\hline Emotional function (1-84) & $40.87(4.15)$ & $59.89(4.33)$ \\
\hline Social function (1-35) & $20.55(4.11)$ & $29.32(3.86)$ \\
\hline Total IBDQ (32-224) & $92.03(24.1)$ & $94.82(24.8)$
\end{tabular}

The higher the scores obtained by the patients, the better the functioning. 


\section{Fatigue, disease activity vs. functioning before and after the surgery}

Table VI presents the correlation between fatigue symptom, disease activity, and patients' functioning before and after the surgical treatment.

Pearson correlation was performed to determine the correlation between fatigue, disease activity, and components of IBDQ scores and total IBDQ.

The analysis confirmed a significant correlation $(p<$ 0.001 ) between fatigue severity and systemic function and social function at the level of $p<0.01$ with other areas for patients with CD before the surgical treatment. Disease activity according to the CAI scale correlated $(p<0.01)$ with all of the areas of the IBDQ scale. Moreover, the correlation was confirmed $(p<0.01)$ for patients with UC in the period before the surgery, between fatigue severity and disease activity (CDAI) and performance according to IBDQ. Correlation between

Table IV. Comparison of patients' functioning before and after surgery

\begin{tabular}{|c|c|c|}
\hline $\begin{array}{l}\text { Inflammatory Bowel } \\
\text { Disease Questionnaire }\end{array}$ & $\begin{array}{l}\text { Crohn's disease } \\
(n=30) \\
\text { Mean (SD) }\end{array}$ & $\begin{array}{l}\text { Ulcerative colitis } \\
\qquad(n=30) \\
\text { Mean (SD) }\end{array}$ \\
\hline \multicolumn{3}{|l|}{ Bowel symptoms (1-70): } \\
\hline Before the surgery & $24.78(3.65)$ & $25.23(3.41)$ \\
\hline After the surgery & $\begin{array}{c}44.18(3.55) \\
F=14.13 \\
p=0.0001\end{array}$ & $\begin{array}{c}53.93(4.23) \\
F=14.22 \\
p=0.0001\end{array}$ \\
\hline \multicolumn{3}{|l|}{ Systemic function (1-35): } \\
\hline Before the surgery & $13.01(2.45)$ & $19.52(3.45)$ \\
\hline After the surgery & $\begin{array}{c}22.11(2.55) \\
F=3.78 \\
p=0.021\end{array}$ & $\begin{array}{c}27.59(3.49) \\
F=3.88 \\
p=0.019\end{array}$ \\
\hline \multicolumn{3}{|c|}{ Emotional function (1-84): } \\
\hline Before the surgery & $39.67(4.65)$ & $40.62(4.23)$ \\
\hline After the surgery & $\begin{array}{c}40.87(4.15) \\
F=4.85 \\
p=0.08\end{array}$ & $\begin{array}{c}59.89(4.33) \\
F=4.35 \\
p=0.02\end{array}$ \\
\hline \multicolumn{3}{|l|}{ Social function (1-35): } \\
\hline Before the surgery & $12.05(4.91)$ & $21.12(3.56)$ \\
\hline After the surgery & $\begin{array}{c}20.55(4.11) \\
F=13.78 \\
p=0.001\end{array}$ & $\begin{array}{c}29.32(3.86) \\
F=14.98 \\
p=0.001\end{array}$ \\
\hline \multicolumn{3}{|l|}{ Total IBDQ (32-224): } \\
\hline Before the surgery & $90.66(21.15)$ & $91.02(20.31)$ \\
\hline After the surgery & $\begin{array}{c}92.03(24.1) \\
F=12.59 \\
p=0.001\end{array}$ & $\begin{array}{c}94.82(24.8) \\
F=14.09 \\
p=0.001\end{array}$ \\
\hline
\end{tabular}

fatigue severity together with disease activity and performance after the surgery for patients with CD and UC was noted in all areas of the IBDQ scale.

\section{Discussion}

Right-sided hemicolectomy (resection of the right part of the colon) with anastomosis of the small intestine and transverse colon is the most frequently performed resection surgical procedure in connection with the typical location of $C D$, i.e. in the terminal part of the small intestine [1]. Our study included eight patients who underwent right-sided hemicolectomy. In other cases of different location, segmental resection of the small intestine or colon is performed. In such cases the principle of removal of parts of the intestine for preservation is applied due to the risk of disease recurrence and the need for re-resection in a different location. Our study included nine patients who underwent bowel resection.

Perianal abscesses and fistulas occur in CD much more often than in UC. The risk of fistula appearance after 20 years of illness is approx. 50\%. Treatment of these changes can be difficult and carries a high risk of recurrence and failure [1]. Therefore, it can lead to lower quality of life and decreased social and occupational activity, often leading to severe depression [9]. In our study 13 patients experienced treatment of anal fistulas.

The range of operational procedures performed with UC is smaller when compared to CD. In cases with complications suspected or diagnosed as bowel cancer the colectomy (excision of the colon while maintaining the rectum), total proctocolectomy, or restorative proctocolectomy (removal of the colon while maintaining the sphincter and creating an intestinal reservoir of small intestine) is performed [1]. In our study 30 of the UC patients underwent restorative proctocolectomy with temporary ileostomy.

Our studies showed that IBD patients who underwent surgery for CD and UC had significantly better functioning (bowel symptoms, systemic, emotional, so-

Table V. Fatigue occurrence according to the FACIT-F scale in the period before and after surgical treatment (mean, SD)

\begin{tabular}{lcc} 
FACIT - F scale & $\begin{array}{c}\text { Crohn's disease } \\
\text { Mean (SD) }\end{array}$ & $\begin{array}{c}\text { Ulcerative colitis } \\
\text { Mean (SD) }\end{array}$ \\
\hline Before surgery & $27.25(13.2)$ & $28.76(11.3)$ \\
\hline After surgery & $16.00(12.6)$ & $14.80(10.6)$ \\
& $F=12.06, p=0.001$ & $F=16.58, p=0.0001$
\end{tabular}

The higher the scores obtained by the patients, the greater the fatigue (max. 52 points). 
Table VI. Correlation between fatigue, disease activity, and patients' functioning before and after surgical treatment

\begin{tabular}{|c|c|c|c|c|c|c|c|c|}
\hline \multirow{3}{*}{$\begin{array}{l}\text { Inflammatory Bowel } \\
\text { Disease Questionnaire }\end{array}$} & \multicolumn{2}{|c|}{ Crohn's disease } & \multicolumn{2}{|c|}{ Ulcerative colitis } & \multicolumn{2}{|c|}{ Crohn's disease } & \multicolumn{2}{|c|}{ Ulcerative colitis } \\
\hline & \multicolumn{4}{|c|}{$\begin{array}{c}\text { Before the surgery } \\
\text { Pearson correlation coefficient } \\
\text { (Rho value) }\end{array}$} & \multicolumn{4}{|c|}{$\begin{array}{c}\text { After surgery } \\
\text { Pearson correlation coefficient } \\
\text { (Rho value) }\end{array}$} \\
\hline & FACIT - F & CAI & FACIT - F & CDAI & $\mathrm{FACIT}-\mathrm{F}$ & CAI & $\mathrm{FACIT}-\mathrm{F}$ & CDAI \\
\hline Bowel symptoms (1-70) & $-0.45^{\star}$ & $-0.47^{\star}$ & $-0.39^{*}$ & $-0.36^{\star}$ & $-0.33^{\star}$ & $-0.36^{*}$ & $-0.35^{\star}$ & $-0.31^{\star}$ \\
\hline Systemic function (1-35) & $-0.57^{\star \star}$ & $-0.43^{\star}$ & $-0.41^{*}$ & $-0.39^{\star}$ & $-0.41^{*}$ & $-0.44^{*}$ & $-0.35^{\star}$ & $-0.38^{*}$ \\
\hline Emotional function (1-84) & $-0.42^{*}$ & $-0.37^{\star}$ & $-0.40^{*}$ & $-0.43^{*}$ & $-0.38^{*}$ & $-0.28^{\star}$ & $-0.36^{*}$ & $-0.35^{\star}$ \\
\hline Social function (1-35) & $-0.56^{\star \star}$ & $-0.48^{*}$ & $-0.45^{\star}$ & $-0.42^{\star}$ & $-0.39^{\star}$ & $-0.36^{\star}$ & $-0.39^{\star}$ & $-0.48^{\star}$ \\
\hline Total IBDQ & $-0.44^{*}$ & $-0.33^{\star}$ & $-0.28^{*}$ & $-0.38^{*}$ & $-0.51^{\star}$ & $-0.37^{\star}$ & $-0.29^{*}$ & $-0.49^{\star}$ \\
\hline
\end{tabular}

${ }^{* *} p<0.001 ;{ }^{*} p<0.01$

cial domains) and higher IBDQ total scores compared to the patients before the surgery.

Similarly, other studies have shown that IBD patients who underwent surgery for CD and UC had significantly higher IBDQ bowel and IBDQ total scores than those who were under long-term medical management [17].

The analysis of literature referring to the occurrence of fatigue indicated that the largest number of publications are dedicated to non-specific inflammatory bowel diseases discussed in terms of pathophysiology, symptoms, diagnosis, or treatment. There are theoretical works, with a particular emphasis on an article by Czuber-Dochan et al. [10,11] about the unification of the term fatigue and its correct use against these disease entities. Because it is a subjective symptom that is difficult to assess objectively, the authors in their publications try to analyse the occurrence of symptom of fatigue by describing the feelings of patients. Patients gave descriptive answers about fatigue [7, 8]. So far, there have not been any published studies concerning the analysis of fatigue in the perioperative or postoperative period. This problem was studied regardless of the surgical treatment, but instead the impact of demographic and clinical determinants were analysed (e.g. prevalence of anaemia, duration of disease).

The results of our study revealed that there is no difference between both disease entities when it comes to fatigue severity. This may be due to the fact that they have similar symptoms, severity of disease, and treatment. It is crucial that fatigue decreases after surgical treatment; because one of the most bothersome symptoms is alleviated, the disease activity decreases - most frequently also the number of bowel movements also decreases. Our research indicates that after the surgical treatment systemic function improved within patients with CD and UC, and bowel ailments decreased.
Romberg-Camps et al. [18] analysed fatigue in patients with non-specific inflammatory bowel diseases, who had not undergone the surgical treatment. The results of their research show that the symptom is present in the remission period as well as during the active course of the disease. Disease duration complications (such as anaemia) are not significant because the fatigue persists all the time. The above-mentioned research indicates that among patients with $C D$ the presence of fatigue did not show differences in fatigue severity regarding sex or age, whereas women with UC presented higher levels of fatigue than men. Similar results were obtained by Simren et al. [19]. It was also found that the pharmacological therapy (corticosteroids, immunosuppressive medications) strengthened the fatigue, compared to patients who were treated with infliximab or did not use drugs at all. The authors in their work also compared the severity of fatigue between the two disease entities, and in contrast to our results they proved that people with CD were more exhausted physically and mentally than patients with UC. They also indicated that, despite the knowledge that in the non-specific bowel diseases the symptom of fatigue is very frequent; however, there are a lack of publications analysing this problem [19]. The other authors also indicated that fatigue can be affected by used medications [20, 21]. Similar data were also obtained in the research by Römkens [22].

Banovica et al. [23] investigated fatigue among subjects with CD. As in the research mentioned above, it appeared that even if the disease is not activated, fatigue is still present. Opheim et al. [24] demonstrated the occurrence of severe fatigue in $39 \%$ of patients with CD and UC. But in patients with CD severe fatigue concerned $43 \%$ of patients, while with UC $33 \%$. 
Sleep disorders can have a great impact on the severity of symptoms. Chronic fatigue can induce sleep disorders and depression [23]. Several studies have concerned the relationship between disease activity and severity of fatigue, indicating that the severity of fatigue correlates with the severity of the disease and treatment. Under the influence of the treatment, the disease activity is reduced, and simultaneously also the degree of fatigue decreases [25]. With the increase of the intensity of fatigue, performance and quality of life of patients is reduced [26]. According to the findings of Norton, poorer quality of life and female gender were the major associations of fatigue in IBD [27].

Several studies have confirmed the relationship between quality of sleep, inflammation, and the severity of fatigue. In one of the studies up to $71 \%$ of patients with IBD reported repetitive awakening mainly due to night diarrhoea or abdominal pain more than 1-2 times/week [27].

An important goal of our research was to determine the relationship between fatigue and functioning of patients, taking into account the activity of the disease.

In our study, in patients with CD before surgical treatment, we indicated the existence of a correlation between severity of fatigue and systemic performance $(p<0.001)$ and social performance, as well as within the other areas at the level of $p<0.01$. Disease activity in CAl scale correlated $(p<0.01)$ with all areas of IBDQ scale.

In patients with UC before the surgical treatment, correlation was indicated $(p<0.01)$ between severity of fatigue and disease activity (CDAI) and performance in accordance with the IBDQ scale. Whereas after the surgical procedure in patients with CD and UC weaker correlation was indicated between severity of fatigue and disease activity and performance in terms of intestine ailments, systemic, emotional, and social performance.

Many studies show the relationship between the severity of fatigue and the activity of the disease [28-32]. These studies show that in IBD, higher levels of fatigue have most frequently been associated with increased disease activity, but also numerous other physical and psychological factors that may influence fatigue (clinical complications, abdominal pain, pharmacological therapy, fear, and depression) [30-32]. In contrast, Cohen's study showed that disease activity was associated with fatigue in CD, but not in UC [33].

It appears that social support, and first of all the daily contact with people, professional work, and the feeling of being needed puts fatigue at a low level. Loneliness and idleness generate a greater sense of weakness and exhaustion, probably mainly psychologically [34]. These studies did not analyse the effect of performing work on the level of fatigue. In agreement with Van Langenberg et al. [2], we believe that further studies should be carried out regarding fatigue in inflammatory bowel diseases, in a larger group of patients, and they should take into consideration the other determinants and continue to examine the impact of surgical treatment on fatigue. Our study is an introduction to the important issue of the occurrence of fatigue in patients with IBD.

\section{Conclusions}

The severity of fatigue in patients with inflammatory bowel diseases is determined as medium. Surgical treatment significantly reduces the fatigue symptom in patients with IBD. The severity of fatigue in the preoperative period is much higher than after performed surgery. The severity of fatigue correlates with disease activity and functioning in the respective areas. There is a need for further research on the assessment of fatigue in inflammatory bowel disease in a larger group of patients, taking into consideration other determinants (socio-demographic factors, disease activity, lifestyle, treatment).

\section{Acknowledgments}

We would like to thank John Coueslant for his linguistic consultation in this paper.

\section{Conflict of interest}

The authors declare no conflict of interest.

\section{References}

1. Bernstein CN, Fried M, Krabshuis JH, et al. World Gastroenterology Organization Practice Guidelines for the Diagnosis and Management of IBD in 2010. Inflamm Bowel Dis 2010; 16: 112-24.

2. Van Langenberg DR, Gibson PR. Systematic review: fatigue in inflammatory bowel disease. Aliment Pharmacol Ther 2010; 32: 131-43.

3. Norton C, Czuber-Dochan W, Bassett P, et al. Assessing fatigue in inflammatory bowel disease: comparison of three fatigue scales. Aliment Pharmacol Ther 2015; 42: 203-11.

4. Minderhoud MI, Oldenburg B, van Dam PS, van Berge Henegouwen GP. High prevalence of fatigue in quiescent inflammatory bowel disease is not related to adrenocortical insufficiency. Am J Gastroenterol 2003; 98: 1088-93.

5. Grimstad T, Norheim KB, Isaksen K, et al. Fatigue in newly diagnosed Inflammatory Bowel Disease. J Crohns Colitis 2015; 20: 725-30.

6. Medical Subject Headings (MESH). National Library of Medicine 2013.

7. Servaes P, Verhagen C, Bleijenberg G. Fatigue in cancer patients during and after treatment: prevalence, correlates and interventions. Eur J Cancer 2002; 38: 27-43. 
8. Swain MG. Fatigue in chronic disease. Clin Sci 2000; 99: 1-8.

9. Vogelaar L, van't Spijker A, Timman R, et al. Fatigue management in patients with IBD: a randomised controlled trial. BMJ 2014; 6: 911-8.

10. Czuber-Dochan W, Ream E, Norton C. Review article: description and management of fatigue in inflammatory bowel disease. Aliment Pharmacol Ther 2013; 37: 505-16.

11. Czuber-Dochan W, Dibley L. The experience of fatigue in people with inflammatory bowel disease: an exploratory study. J Adv Nur 2013; 69: 1987-99.

12. Bączyk G, Formanowicz D, Gmerek $九$, Krokowicz P. Health-related quality of life assessment among patients with inflammatory bowel diseases after surgery - review. Gastroenterology Rev 2017; 12: 6-16.

13. Irvine E J. Quality of life - measurement in inflammatory bowel disease. Scand J Gastroenterol 1993; 199: 22-7.

14. Tinsley A, Macklin EA, Korzenik, JR Sands BE. Validation of the Functional Assessment of Chronic Illness Therapy-Fatigue (FACIT-F) in patients with inflammatory bowel disease. Aliment Pharmacol Ther 2011; 34: 1328-36.

15. Best WR, Becktel JM, Singleton JW. Rederived values of the eight coefficients of the Crohn's Disease Activity Index (CDAI) Gastroenterology 1979; 77: 843-6.

16. Hodgson HJ, Bhatti M. Assessment of disease activity in ulcerative colitis and the Cronh's disease. Inflamm Bowel Dis 1995; 1: 117-43.

17. Subasinghe D, Navarathna NMM, Samarasekera DN. Faecal incontinence and health related quality of life in inflammatory bowel disease patients: findings from a tertiary care center in South Asia. World J Gastrointest Pharmacol Ther 2016; 7: 447-52.

18. Romberg-Camps MJL, Boy Y. Fatigue and health-related quality of life in inflammatory bowel disease: results from a population-based study in the Netherlands: The IBD-South Limburg cohort. Inflamm Bowel Dis 2010; 16: 2137-47.

19. Simren M, Svedlund J, Posserud I, et al. Predictors of subjective fatigue in chronic gastrointestinal disease. Aliment Pharmacol Ther 2008; 28: 638-47.

20. Jelsness-Jorgensen LP, Bernklev TB, Henriksen M, et al. Chronic fatigue is associated with impaired health-related quality of life in inflammatory bowel disease. Aliment Pharmacol Ther 2011; 33: 106-14.

21. Lee TWT, Iser JH, Sparrow MP, et al. Thiopurines, a previously unrecognised cause for fatigue in patients with inflammatory bowel disease. J Crohns Colitis 2009; 3: 196-9.

22. Römkens TEH. High prevalence of fatigue in inflammatory bowel disease: a case control study. J Crohns Colitis 2011; 5: 332-7.

23. Banovica I, Gilibertb D, Cosnesc J. Crohn's disease and fatigue: constancy and co-variations of activity of the disease, depression, anxiety and subjective quality of life. Psychol Health Med 2010; 15: 394-405.

24. Opheim R, Fagermoen MS, Bernklev T, et al. Fatiuge interference with daily living among patients with inflammatory bowel disease. Qual Life Res 2014; 23: 709-19.

25. Joyce JC, Waljee AK, Khan T, et al. Identification of symptom domains in ulcerative colitis that occur frequently during flares and are responsive to changes in disease activity. Health Qual Life Outcomes 2008; 6: 69.
26. Graff LA, Walker JR, Lix L, et al. The relationship of inflammatory bowel disease type and activity to psychological functioning and quality of life. Clin Gastroenterol Hepatol 2006; 4: 1491501.

27. Norton C, Czuber-Dochan W, Bassett P, et al. Assessing fatigue in inflammatory bowel disease: comparison of three fatigue scales. Aliment Pharmacol Ther 2015; 42: 203-11.

28. Ranjbaran Z, Keefer L, Farhadi A, et al. Impact of sleep disturbances in inflammatory bowel disease. J Gastroenterol Hepatol 2007; 22: 1748-53.

29. Graff LA, Vincent N, Walker JR, et al. A population based study of fatigue and sleep difficulties in inflammatory bowel disease. Inflamm Bowel Dis 2011; 17: 1882-9.

30. Jelsness-Jřrgensen LP, Bernklev T, Henriksen M, et al. Chronic fatigue is more prevalent in patients with inflammatory bowel disease than in healthy controls. Inflamm Bowel Dis 2011; 17: 1564-72.

31. Abdalla MI, Sandler RS, Kappelman MD, et al. The impact of ostomy on quality of life and functional status of Crohn's disease patients within CCFA partners. Inflamm Bowel Dis 2016; 22: 2658-64.

32. Vogelaar L, de Haar C, Aerts BR, et al. Fatigue in patients with inflammatory bowel disease is associated with distinct differences in immune parameters. Clin Exp Gastroenterol 2017; 10: 83-90.

33. Cohen BL, Zoëga $H$, Shah SA, et al. Fatigue is highly associated with poor health-related quality of life, disability and depression in newly-diagnosed patients with inflammatory bowel disease, independent of disease activity. Aliment Pharmacol Ther 2014; 39: 811-2.

34. Hauser W, Stallmach A, Kocalevent RD, et al. Biopsychosocial predictors of fatigue in quiescent and mild ulcerative colitis: an explorative study. GMS Psycho Social Med 2005; 2: Doc07.

Received: 25.04 .2018

Accepted: 22.02.2019 